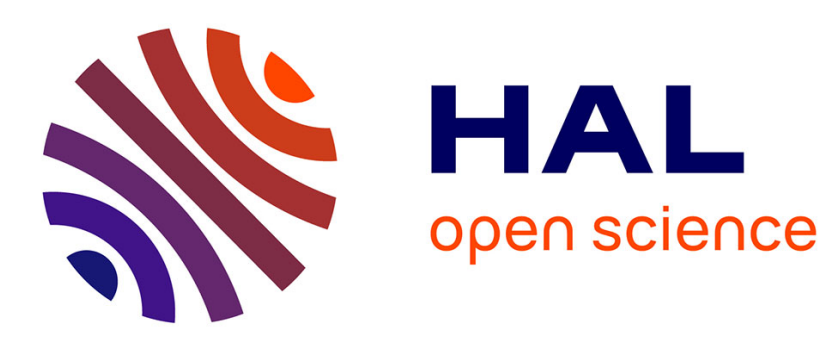

\title{
How Does Seismic Rupture Accelerate? Observational Insights From Earthquake Source Time Functions
}

\author{
Julien Renou, Martin Vallée, Pierre Dublanchet
}

\section{To cite this version:}

Julien Renou, Martin Vallée, Pierre Dublanchet. How Does Seismic Rupture Accelerate? Observational Insights From Earthquake Source Time Functions. Journal of Geophysical Research: Solid Earth, 2019, 124 (8), pp.8942-8952. 10.1029/2019JB018045 . hal-02367631

\section{HAL Id: hal-02367631 \\ https://hal.science/hal-02367631}

Submitted on 6 Aug 2020

HAL is a multi-disciplinary open access archive for the deposit and dissemination of scientific research documents, whether they are published or not. The documents may come from teaching and research institutions in France or abroad, or from public or private research centers.
L'archive ouverte pluridisciplinaire HAL, est destinée au dépôt et à la diffusion de documents scientifiques de niveau recherche, publiés ou non, émanant des établissements d'enseignement et de recherche français ou étrangers, des laboratoires publics ou privés. 


\author{
RESEARCH ARTICLE \\ 10.1029/2019JB018045 \\ Key Points: \\ - Source time functions exhibit a \\ magnitude-independent signal \\ during periods of monotonically \\ increasing moment rate \\ - Moment rate time evolution in the \\ development phase does not follow \\ the steady quadratic self-similar \\ growth \\ - Rupture models with variable \\ rupture velocity and/or stress \\ drop are required to reproduce the \\ observations
}

Supporting Information:

- Supporting Information S1

Correspondence to:

J. Renou,

renou@ipgp.fr

Citation:

Renou, J., Vallée, M., \& Dublanchet, P. (2019). How does seismic rupture accelerate? Observational insights from earthquake source time functions. Journal Geophysical Research: Solid Earth, 124, 8942-8952. https://doi.org/10.1029/2019JB018045

Received 16 MAY 2019 Accepted 11 AUG 2019

Accepted article online 15 AUG 2019 Published online 24 AUG 2019

(C)2019. American Geophysical Union. All Rights Reserved.

\section{How Does Seismic Rupture Accelerate? Observational Insights From Earthquake Source Time Functions}

\author{
Julien Renou ${ }^{1}$ iD, Martin Vallée ${ }^{1}$, and Pierre Dublanchet ${ }^{2}$ iD \\ ${ }^{1}$ Université de Paris, Institut de physique du globe de Paris, CNRS, Paris, France, ${ }^{2}$ MINES ParisTech, PSL Research \\ University, Centre de Géosciences, Fontainebleau, France
}

\begin{abstract}
Observation of the seismic process for a large earthquake population is of key interest to detect potential magnitude-dependent behaviors and, more generally, to quantify how seismic rupture develops. In contrast with studies focusing on the first radiated waves, we here propose to characterize the growing phase leading to the main seismic moment release episode(s), which we refer to as the development phase. Our analysis uses the 2,221 teleseismic source time functions (STFs) of shallow dip-slip earthquakes provided by the global SCARDEC database and consists in measuring the moment acceleration during the development phase at prescribed moment rates. This approach is therefore insensitive to hypocentral time uncertainties and aims at quantifying how seismic ruptures accelerate, independently of when they accelerate. Our results first show that rupture acceleration does not exhibit any magnitude-dependent signal emerging above the intrinsic measurements variability. We thus use the full STF catalog to characterize the moment rate $\dot{M}_{d}$ of the development phase and show that, on average, $\dot{M}_{d}(t) \propto t^{n_{d}}$ with $n_{d}$ equal to 2.7. This time evolution therefore does not follow the steady $t^{2}$ growth expected for classical circular crack models, which indicates that stress drop and/or rupture velocity transiently vary during the development phase. We finally illustrate with a synthetic STF catalog that, due to initial rupture variability, approaches based on hypocentral time are not expected to fully characterize the behavior of the development phase.
\end{abstract}

\section{Introduction}

The mechanisms governing the seismic rupture expansion and giving rise to earthquakes of very different magnitudes remain debated. From an observational point of view, past studies most often focused on the first seismic signals radiated by the earthquake rupture, with the goal to provide useful information for early warning. Several studies (Beroza \& Ellsworth, 1996; Colombelli et al., 2014; Olson \& Allen, 2005) argued for the existence of a magnitude-dependent initial signal, connecting the early phases of the rupture process with its final magnitude. The existence of such a signal could be explained, for example, if an earthquake is more likely to become a large one if its initial phase occurs in rupture-prone areas. Large earthquakes would then start differently from small ones, at least in a statistically predictable way. Conversely, many rupture onsets have been observed without detecting any clues related to the final earthquake magnitude; seismic rupture is then interpreted as a "self-similar" process, meaning that large earthquakes are only upscaled versions of small-magnitude events, without having their own characteristics (Aki, 1967). As a result of this concept, studies showed, for instance, that stress drop and rupture velocity are independent of the magnitude, or that the seismic moment is proportional to the cube of the earthquake duration (Allmann \& Shearer, 2009; Kanamori \& Anderson, 1975). The self-similar behavior can be reproduced by a cascade model, in which the rupture starts from a very small patch, which size is undetectable by seismological investigation. Then rupture grows in a self-similar way, implying that the final magnitude is controlled by the earthquake duration. Such behavior has been for example observed by Uchide and Ide (2010) in their analysis of earthquakes in the Parkfield area.

Observations of the earthquake process, however, reveal that real ruptures frequently depart from such simple models and that the peak moment rate can be reached after a nonmonotonical or delayed process. Studying how rupture behaves when entering into its most active phase (that we hereafter refer to as the "development phase") therefore requires an analysis of the whole process and not only its beginning. To do so, we propose to make use of the large catalog of moment rate functions (or source time functions, STFs) provided by the SCARDEC database (Vallée \& Douet, 2016). SCARDEC database has first been used 
to extract global source properties, such as source-averaged stress/strain drop or rupture velocity (Courboulex et al., 2016; Chounet et al., 2017; Chounet \& Vallée, 2018; Vallée, 2013) and is now more and more exploited to characterize the transient parts of STFs (Meier et al., 2017; Melgar \& Hayes, 2017; 2019). With a similar objective as the studies based on the early stages of the rupture, we will first explore if the moment acceleration in the development phase correlates with the magnitude of the event. We will then characterize the temporal moment evolution of this specific phase, in order to provide observational constraints on rupture propagation models. We finally further illustrate, with a realistic synthetic STF catalog, why the characteristics of the development phase are difficult to retrieve from the study of the early rupture stages.

\section{Moment Acceleration in the Development Phase} 2.1. SCARDEC STF Database and Earthquake Development Phase

Exhaustive catalogs of STFs (describing the time evolution of the moment rate $\dot{M}$ ) can be built with two distinct methods which both use teleseismic data from the Federation of Digital Seismograph Network. The first approach determines a finite fault model of the seismic source (in general for earthquakes with $M_{w}>7$; Hayes, 2017; Ye et al., 2016) from which the absolute STF is computed. On the other hand, in the SCARDEC method (Vallée et al., 2011), seismic moment, focal mechanism, source depth, and STFs are more directly obtained through a deconvolution process (see also Tanioka, 1997). At each station and for each phase $(P$ or $S$ ), apparent source time functions (ASTFs) are extracted, whose shapes differ due to space-time source effects (Chounet et al., 2017). In order to take into account both this expected distorsion and possible outliers (due to nodal radiation, incorrect instrument response, etc.), SCARDEC database (Vallée \& Douet, 2016) provides two representative STFs for each event. A mean STF is first obtained by correlating in time all $P$ wave ASTFs (less sensitive to space-time source effects than $S$ wave ASTFs), removing ASTFs far from the beam, and averaging the remaining ASTFs. The optimal STF is then chosen as the $P$ wave ASTF, which is the closest to the mean STF. Such an optimal STF is unlikely to be among the most distorted ASTFs, and its shape is not affected by the smoothing present in the mean STF. The optimal STFs are therefore considered in this study. Deep ( $>70 \mathrm{~km})$ and pure strike-slip events are removed from the database due to their specific behavior (Houston, 2001) and the difficulty to robustly extract their $P$ wave STFs, respectively. The catalog is finally composed of 2,221 earthquake STFs (from 1992 to 2017), whose magnitudes range from $M_{w} 5.5$ to $M_{w} 9.1$ (2011 Tohoku earthquake) and durations from 2 to $120 \mathrm{~s}$.

We aim here at isolating the development phase, that is, the time period where STFs grow toward their peak moment rate $F_{m}$ (that they reach at time $T_{m}$ ). Taking into account that the moment rate always flattens before reaching $F_{m}$, we do not consider the highest STF values to be part of the development phase: in the following, we only select the parts of the STF which are before $T_{m}$, and whose values are below $0.7 F_{m}$. At low moment rate values, we would ideally track the development phase from its very beginning. However, SCARDEC STFs are retrieved by deconvolving the full P-waveform (under physical constraints such as STF positivity), and the STF fidelity at values much lower than $F_{m}$ is therefore expected to be relatively low. As a result, we do not analyze here the development phase for STF values lower than $0.07 F_{m}$. The value of these two selected lower and upper limits are not critical and other choices (e.g., starting at $0.05 F_{m}$ and stopping at $0.5 F_{m}$ ) do not affect significantly the following results (see Figure S13 in the supporting information).

In order to isolate the development phases in all cases, we consider the two following possible configurations of STFs. The simplest and most common case (representing 62\% of the STF catalog) is illustrated by the STFs shown in Figures 1a and 1b. Here, even when the STF does not grow monotonically toward its peak, there is a unique monotonic domain connecting the values between $0.07 F_{m}$ and $0.7 F_{m}$. This specific section of the STFs, shown in red in Figure 1, is selected as the development phase. STFs with complex shapes however do not have such a unique monotonic domain (Figure 1c). In this case, we work on the time interval defined by two times $T_{0}$ and $T_{1}: T_{0}$ is the latest time preceding $T_{m}$ when the STF is as low as $0.07 F_{m}$ and $T_{1}$ is the latest time preceding $T_{m}$ when the STF is not above $0.7 F_{m}$. In the $\left[T_{0} T_{1}\right]$ interval, there may be several local maxima $F_{p}(p=1, P)$, around which rupture is not considered to be in a development phase. The development phase is then selected as the combination of monotonic phases preceding each $F_{p}$, from the time when they exceed the largest value of all the preceding local maxima (or from $T_{0}$ if $p=1$ ) to the time where they reach $0.7 F_{p}$. As a consequence, if one of the local peak values before $F_{p}$ is larger than $0.7 F_{p}$, the monotonic phase preceding $F_{p}$ is not considered. We finally select the monotonic phase up to $T_{1}$ (from the time where the STF reaches $F_{P}$, or from $T_{0}$ if $P=0$ ). In these complex cases, the development phase is therefore the combination of at most $(P+1)$ growing sections of the STFs. 


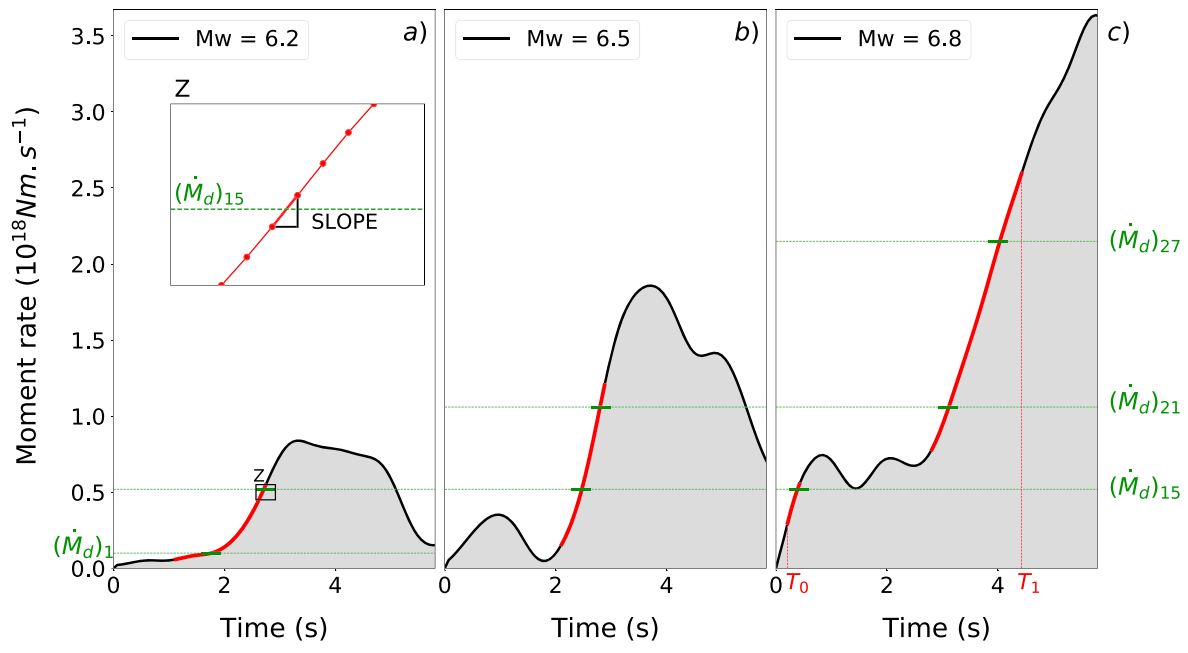

Figure 1. Examples of extraction of the development phase (in red) for representative STF shapes. In (a) and (b), STFs have a monotonic section connecting the values between $0.07 F_{m}$ and $0.7 F_{m}$. Panel (c) is an example of STF with complex shape in which development phase is extracted in the $\left[T_{0} T_{1}\right]$ time interval (see section 2.1). These three illustrative events have different magnitudes and their development phases start at different times. Moment acceleration is computed at the time where the development phase crosses the prescribed moment rates $\left(\dot{M}_{d}\right)_{i}$, if this intersection exists. Four examples of $\left(\dot{M}_{d}\right)_{i}$ values (for $i=1,15,21$, and 27) are shown in green. The sampling rate is equal to $0.07 \mathrm{~s}$. Note that the approximate reference time shown in the bottom of each STF is not used in this approach.

According to the aforementioned definitions, the development phase may be delayed compared to hypocentral time, meaning that we do not intend to characterize the earliest signals emitted by the seismic rupture. This approach therefore differs from studies specifically analyzing the latter signals in order to explore the concept of earthquake determinism since the earthquake initiation (Meier et al., 2017; Melgar \& Hayes, 2017, 2019).

\subsection{Seismic Moment Acceleration Within the Development Phase}

Once the development phase is extracted for each STF, we aim at characterizing it without using hypocentral time information, in order to quantify how rupture develops independently of when rupture develops. Formally, we look for the moment evolution of the development phase $M_{d}$ where $M_{d}(t)=M\left(t+T_{d}\right), T_{d}$ being the unknown time at which the development phase starts. A way to characterize $M_{d}$ is to consider a discrete sampling of prescribed moment rates $\left(\dot{M}_{d}\right)_{i}$, and to compute the seismic moment acceleration (STF slope) each time that the development phase crosses $\left(\dot{M}_{d}\right)_{i}$. To do so, we consider 40 different values of $\left(\dot{M}_{d}\right)_{i}(i=1,40)$, from $10^{17}$ to $10^{19} \mathrm{Nm} / \mathrm{s}$, in order to sample the development phase of most earthquakes. Outside of this range, moment rates are either mostly below $0.07 F_{m}$ or above $0.7 F_{m}$ and cross only a few development phases. As further documented later, the maximum considered moment rate $\left(10^{19} \mathrm{Nm} / \mathrm{s}\right)$ is typically reached $6 \mathrm{~s}$ after the beginning of the development phase for monotonically growing STFs. In terms of magnitude, the smallest earthquakes of the SCARDEC database $\left(M_{w}=5.5\right)$ can be analyzed by this sampling, and only the largest earthquakes $\left(M_{w}>8.4\right)$ are systematically excluded. Figure 1 illustrates the method for three STFs and four moment rates $\left(\dot{M}_{d}\right)_{i}$ (green dashed lines). Low values of moment rate are mostly sampled by small events (as they will lie below $0.07 F_{m}$ for large ones) and high values of moment rate are mostly sampled by large events (as they will lie above $0.7 F_{m}$ for small ones). However, this general behavior does not prevent us from sampling a large range of magnitudes at a given moment rate. As shown in the example of Figure 1, the moment acceleration of the development phase at the $\left(\dot{M}_{d}\right)_{15}$ level can be computed from $M_{w}=6.2$ to $M_{w}=6.8$.

\subsection{Variability and Magnitude-Independent Behavior}

Such slope measurements can be first used to detect a potential magnitude-dependent behavior, in which the slope measured when the development phase crosses prescribed moment rates would be for instance steeper for larger events. For the $N_{i}$ development phases crossing $\left(\dot{M}_{d}\right)_{i}$, we compute the slope values $\left(\ddot{M}_{d}\right)_{i j}(j=$ $\left.1, N_{i}\right)$ as a function of $M_{w}$, to observe whether or not a magnitude-dependent signal appears. Figure 2 shows an example of the $892\left(\ddot{M}_{d}\right)_{15 j}$ values for $\left(\dot{M}_{d}\right)_{15}=5.2 \times 10^{17} \mathrm{Nm} / \mathrm{s}$. The following analysis of $\ddot{M}_{d}$ values with respect to $M_{w}$ has to be done with care, because a given $\left(\dot{M}_{d}\right)_{i}$ value does not sample equally well all 


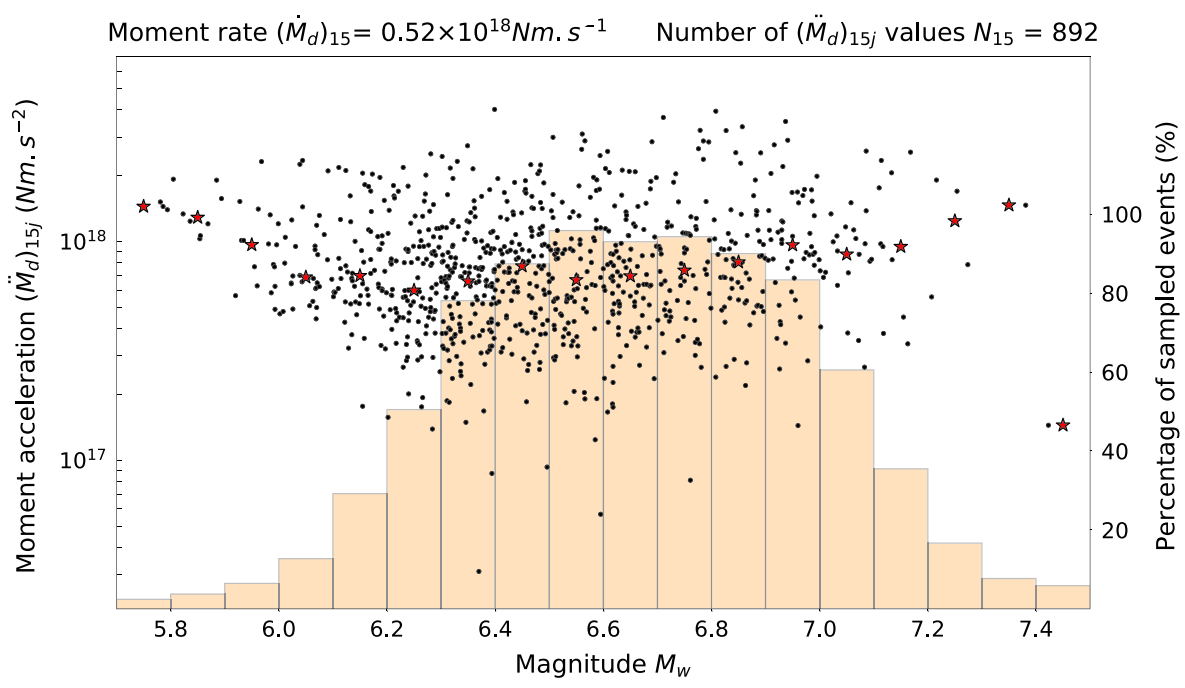

Figure 2. Moment acceleration as a function of magnitude for the prescribed moment rate of $5.2 \times 10^{17} \mathrm{Nm} / \mathrm{s}\left(\left(\dot{M}_{d}\right)_{15}\right)$. The filled histogram represents the ratio (\%; see scale to the right) of sampled events in each $M_{w}$ bin. Comparisons between moment acceleration and magnitude can be safely done when almost all the source time functions of a given magnitude are sampled ( $>80 \%$ ), here between $M_{w}=6.3$ and $M_{w}=7.0$. Red stars are median values for each magnitude bin. Similar figures for all the prescribed moment rates are provided in Figures S3-S12.

magnitude ranges (section 2.2), as also illustrated in Figure 2: the histogram shows the ratio of sampled STFs in each $M_{w}$ bin, and this value decreases both toward low $M_{w}$ (only impulsive STFs reach $\left.\left(\dot{M}_{d}\right)_{i}\right)$ and toward high $M_{w}$ (only STFs with relatively low $F_{m}$ have $\left(\dot{M}_{d}\right)_{i}$ in their development phase). As a consequence, $\left(\ddot{M}_{d}\right)_{i j}$ values are expected to be biased toward high values for small-magnitude events, as confirmed by Figure 2. We thus focus on the $M_{w}$ domain where most of the development phases cross the chosen $\left(\dot{M}_{d}\right)_{i}$ (for example between $M_{w}=6.3$ and $M_{w}=7.0$ in the case shown in Figure 2).

Figure 2 does not exhibit any clear dependency between $\left(\ddot{M}_{d}\right)_{15 j}$ and $M_{w}$, and the same behavior is observed for all the other prescribed $\left(\dot{M}_{d}\right)_{i}$ (Figures S3 to S12 in the supporting information). This shows that if a magnitude-dependent signal exists, it is fully dominated by the intrinsic variability of the development phase. This means that when an earthquake develops and reaches a given moment rate $\left(\dot{M}_{d}\right)_{i}$, moment acceleration cannot be used as an indicator of the final magnitude (only a lower bound can of course be estimated based on the seismic moment already released). This observation may appear different from the recent results of Melgar and Hayes (2019), who extracted a magnitude-dependent signal from STF accelerations (using also the SCARDEC catalog). Their approach is however fundamentally different as they simply computed an averaged moment acceleration by dividing the moment rate from the rupture time, at several prescribed rupture times $\tau(\tau=2,5,10,20 \mathrm{~s})$. Using this definition, they observe an increase of the moment acceleration with the final event magnitude, clearly appearing for $\tau$ equal to 10 and $20 \mathrm{~s}$. In such an analysis, there is, however, no guarantee that the earthquake at $\tau$ is still in its development phase, particularly when $\tau$ is a significant fraction of the global earthquake duration. As an example, $20 \mathrm{~s}$ is a significant fraction of the global duration of an $M_{w}=8$ earthquake (whose average global duration is about $60 \mathrm{~s}$, e.g., Vallée, 2013). It is therefore not uncommon, at $20 s$, that $M_{w}=8$ earthquakes STFs flatten as they approach their peak moment rate (and some of them may have already passed it). As a result, on average, acceleration can be understood to be statistically lower than for a $M_{w}=9$ earthquake, for which the peak always occurs far after 20 s. Melgar and Hayes (2019) results likely reflect the magnitude-dependent shape of the earthquake STFs, at a macroscopic scale, while we are here specifically studying their fast growing parts.

\section{Time Evolution of the Development Phase}

\subsection{Observational Evidence of a Power Law Between $\ddot{M}$ and $\dot{M}$}

The magnitude independency derived in the previous section justifies the combined use of $\left(\ddot{M}_{d}\right)_{i j}$ for all values of $i$, in order to determine a generic behavior of the rupture development. Figure 3 represents (in $\log$-log scale) all the moment acceleration values as a function of the moment rate values (yellow dots). Direct observation in Figure 3 reveals that $\ddot{M}_{d}$ grows with $\dot{M}_{d}$, which first implies that the time evolution 


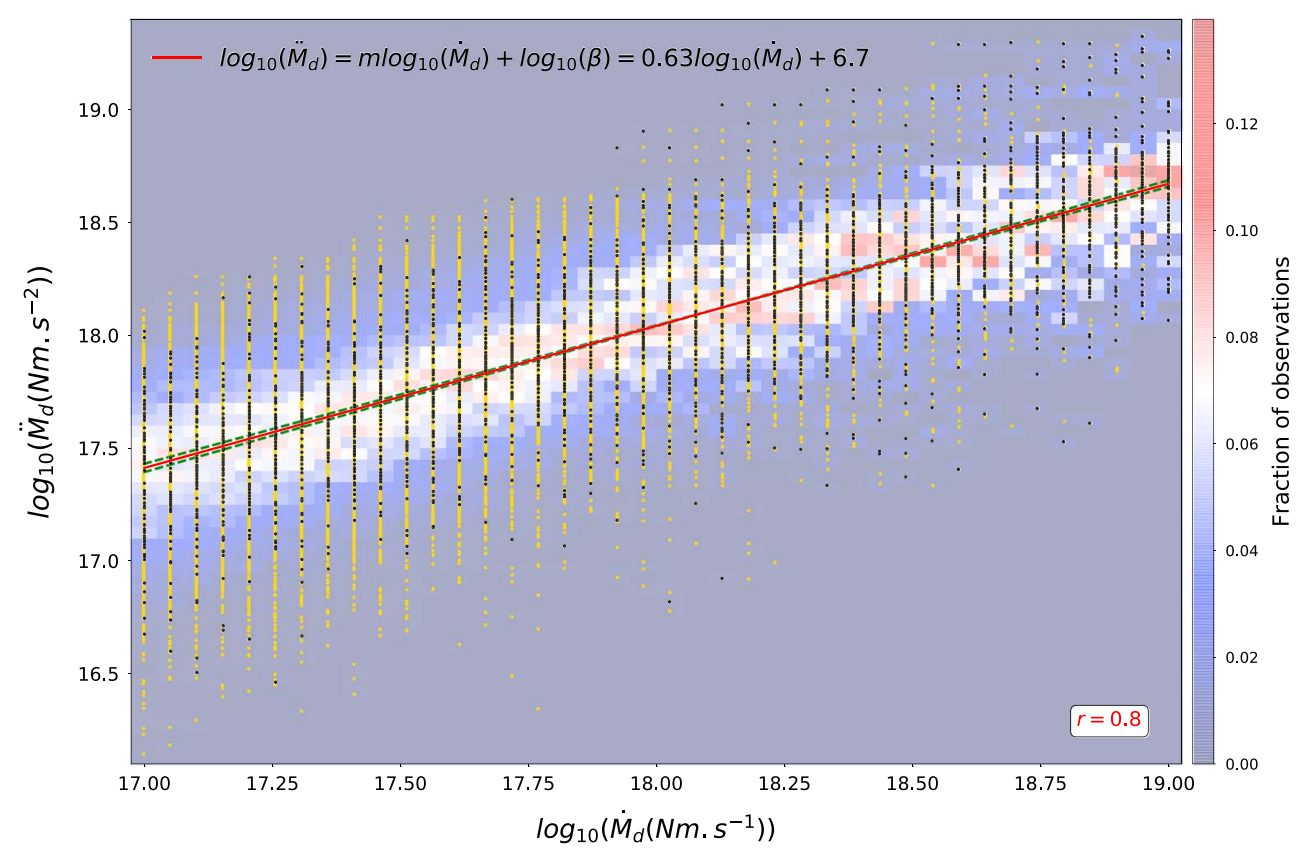

Figure 3. Moment acceleration $\ddot{M}_{d}$ as a function of moment rate $\dot{M}_{d}(\log -\log$ scale). Each yellow dot corresponds to an individual $\left(\ddot{M}_{d}\right)_{i j}$ value, and black dots are 75 randomly selected values for each $\left(\dot{M}_{d}\right)_{i}$ used to compute the linear fit (see Text S1 in the supporting information). Red line is the best linear fit explaining the data, and its equation and correlation coefficient $r$ are given in the figure. Green dashed lines are fits with extremal values of $m$ and $\log (\beta)$ at the $90 \%$ confidence interval. Background color represents the number of $\left(\ddot{M}_{d}\right)_{i j}$ values normalized by $N_{i}$ for each $\left(\dot{M}_{d}\right)_{i}$. This fraction of observations is computed between $\log \left(10^{15}\right) \mathrm{Nm} / \mathrm{s}^{2}$ and $\log \left(10^{20}\right) \mathrm{Nm} / \mathrm{s}^{2}$ with 100 bins.

of the moment rate in the development phase cannot be linear. In order to quantify the general behavior, we try to fit our observations with a power law of the type $\ddot{M}_{d}=\beta \dot{M}_{d}^{m}$. Using the method detailed in Text S1 in the supporting information, a linear fit (in log-log scale) leads to values of $m=0.63 \pm 0.015$ and $\log (\beta)=6.7 \pm 0.28$ at the $90 \%$ confidence interval, with a correlation coefficient of 0.8 (Figure 3 ).

\subsection{Power Law Time Exponent of the Development Phase}

Analytical models of rupture dynamics (Dahlen, 1974; Kostrov, 1964; Madariaga, 1976; Nielsen \& Madariaga, 2003) have shown that self-similar circular growth with constant stress drop $\Delta \sigma$ and rupture velocity $V_{r}$ leads to a moment rate function of the form $\dot{M}(t)=\alpha t^{n}$ with $n=2$. In this model, the local slip $u$ and slip rate $\dot{u}$ have the shape, in the general case of a time-varying rupture velocity $v_{r}(t)$ :

$$
\begin{gathered}
u(r, t)=\frac{\Delta \sigma}{\mu} \sqrt{a^{2}(t)-r^{2}} \\
\dot{u}(r, t)=\frac{\Delta \sigma}{\mu} v_{r}(t) \frac{a(t)}{\sqrt{a(t)^{2}-r^{2}}}
\end{gathered}
$$

where $\mu$ is the rigidity and $a(t)=\int_{0}^{t} v_{r}(u) \mathrm{d} u$ is the radius of the rupture at time $t$. The moment time evolution therefore follows the following law:

$$
\begin{gathered}
M(t)=2 \pi \mu \int_{0}^{a(t)} u(r, t) r \mathrm{~d} r \\
=\frac{2 \pi}{3} \Delta \sigma\left[\left(a^{2}(t)-r^{2}\right)^{3 / 2}\right]_{a(t)}^{0} \\
=\frac{2 \pi}{3} \Delta \sigma a^{3}(t) .
\end{gathered}
$$


And if $v_{r}(t)=V_{r}$ is constant, $a(t)=V_{r} t$ and we have

$$
\begin{aligned}
& M(t)=\frac{2 \pi}{3} \Delta \sigma V_{r}^{3} t^{3} \\
& \dot{M}(t)=2 \pi \Delta \sigma V_{r}^{3} t^{2} .
\end{aligned}
$$

This quadratic dependency with time is also found from the seismic moment $M_{0}$, by using that $\Delta \sigma=c \mu \frac{\Delta U}{L}$, $M_{0}=\mu \Delta U S$ (where $c$ is a constant, $\Delta U$ the average displacement, $L$ the characteristic dimension of the fault, and $S$ its surface), and considering a seismic rupture growing in a bidimensional way with constant rupture velocity. At the time of the end of the rupture, we have $M_{0} \propto \Delta \sigma V_{r}^{3} T^{3}$ (with $T$ being the final rupture duration), but due to self-similarity, this relation also holds for the moment function $M$ at any earlier time $t$ :

$$
M(t) \propto \Delta \sigma V_{r}^{3} t^{3} \quad \text { leading to } \quad \dot{M}(t) \quad \propto \quad \Delta \sigma V_{r}^{3} t^{2} .
$$

The previously obtained $\ddot{M}_{d}$ values do not directly constrain the time evolution of the development phase, because we only know them as a function of $\dot{M}_{d}\left(\ddot{M}_{d}=\beta \dot{M}_{d}^{m}\right.$, with $\log (\beta)$ and $m$ found equal to $6.7 \pm 0.28$ and $0.63 \pm 0.015$ at the $90 \%$ confidence interval, respectively). However, reorganizing this power law equation, and integrating over time leads to

$$
\int_{0}^{t} \frac{\ddot{M}_{d}(u)}{\left(\dot{M}_{d}(u)\right)^{m}} \mathrm{~d} u=\int_{0}^{t} \beta \mathrm{d} u \quad\left(\forall t>0, \dot{M}_{d}(t)>0\right),
$$

with $t$ being a time inside the development phase. The lower bound of integration in (7) assumes that the observed power law between $\ddot{M}$ and $\dot{M}$ holds from the beginning of the development phase, which appears reasonable because no deviation appears at low moment rates in Figure 3. As $m$ is observationally strictly smaller than 1 (even the extreme $m$ values shown in Figure S1a in the supporting information are strictly smaller than 1), equation (7) has the following solution:

$$
\frac{\left(\dot{M}_{d}(t)\right)^{1-m}}{1-m}=\beta t
$$

where we use the physical constraint $\dot{M}_{d}(0)=0$. The moment rate function can then be rewritten as a function of time:

$$
\dot{M}_{d}(t)=(\beta(1-m))^{\frac{1}{1-m}} \times t^{\frac{1}{1-m}}
$$

In the following, we now define $\alpha_{d}=(\beta(1-m))^{\frac{1}{1-m}}$ and $n_{d}=\frac{1}{1-m}$. Using the values of $m$ and $\beta$, the numerical expression for the time evolution of the development phase is

$$
\dot{M}_{d}(t)=\alpha_{d} \times t^{n_{d}}=10^{16.9 \pm 0.1} \times t^{2.7 \pm 0.11}
$$

where uncertainties for $n_{d}$ and $\alpha_{d}$ correspond to the $90 \%$ confidence intervals estimated in Figure S1b in the supporting information.

In Figure 4, we show this temporal evolution of $\dot{M}_{d}$ and indicate the time window between $\sim 1$ and $\sim 6 \mathrm{~s}$ (corresponding to $\dot{M}_{d}$ values between $10^{17}$ and $10^{19} \mathrm{Nm} / \mathrm{s}$ ), where the shape of $\dot{M}_{d}$ is directly constrained by the observations. As $n_{d}$ is robustly larger than 2, equation (10) indicates that the rupture process during the development phase grows with time with a higher exponent than what the classical self-similar equations (3.2) and (6) predict.

\subsection{Implications for Earthquake Source Physics}

Our results highlight that when seismic rupture efficiently develops, it does not steadily follow the classical $t^{2}$ law predicted by classical self-similar equations. While this simple law is seismologically observed when considering the whole rupture duration $T$ (i.e. $M_{0} \propto T^{3}$ (Bilek et al., 2004; Chounet \& Vallée, 2018; Houston et al., 1998; Vallée, 2013)), it is transiently not respected during the development phase. Such breaks in scaling laws have been recently found by other authors (Archuleta \& Ji, 2016; Denolle \& Shearer, 2016). As their spectral observations are not explained by a self-similar Brune (1970) source spectrum with a single corner frequency, they also suggest the existence of a second timescale related to a transient accelerating phase. 


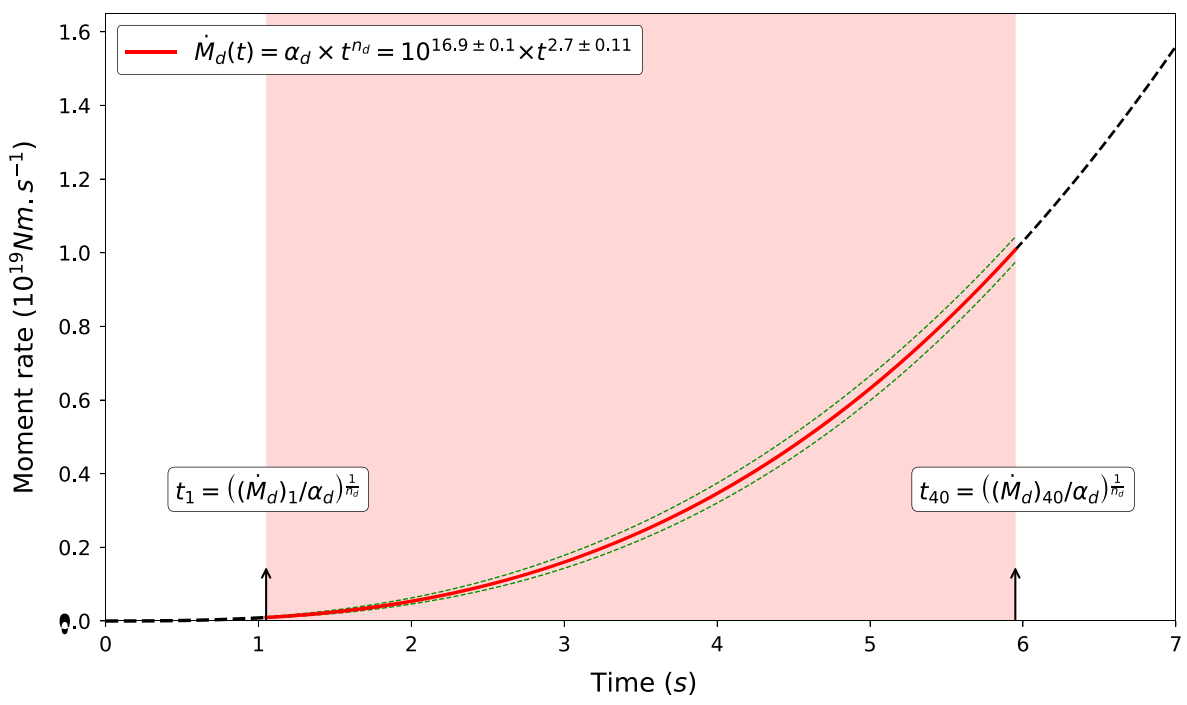

Figure 4. Time evolution of the development phase extracted from the observed power law between $\ddot{M}_{d}$ and $\dot{M}_{d} \cdot t_{1}$ and $t_{40}$ are the times corresponding to the extremal moment rate values $\left(\left(\dot{M}_{d}\right)_{1}=10^{17} \mathrm{Nm} / \mathrm{s}\right.$ and $\left.\left(\dot{M}_{d}\right)_{40}=10^{19} \mathrm{Nm} / \mathrm{s}\right)$ at which the moment accelerations are computed. This time window between $t_{1}$ and $t_{40}$, where the time exponent of the moment rate $n_{d}=2.7$ directly comes from the observations, is highlighted by the red shaded area. Green dashed curves are the extremal curves inferred from $\alpha_{d}$ and $n_{d}$ uncertainties.

As the $t^{2}$ law directly comes from the shape of the slip function in equation (2) and from the constant stress drop and rupture velocity hypotheses, at least one of these assumptions should not be respected during the development phase. The radial model can for example be questioned based on numerical dynamic and kinematic studies (Beroza \& Spudich, 1988; Das \& Kostrov, 1983; Dunham et al., 2003; Zhang et al., 2012) showing that the main asperity may break inward after being encircled by the rupture front. But while this process is expected to generate a large transient moment acceleration, it is less clear how it can reproduce a power law similar to what we observe. If remaining in a radial model with constant rupture velocity, we can also easily derive that a slip function of the form $u(r, t) \propto t^{n_{d}-2} \sqrt{a^{2}(t)-r^{2}}$ would lead to our observed moment rate evolution of the development phase. Such a model implies that stress drop inside the main asperities grows with time (consistent with some studies showing a positive correlation between peak stress drop and magnitude (Causse et al., 2013; Mai et al., 2006)), but as a consequence, it is unlikely that the slip law can be physically written in this case under a simple form similar to equation (2).

An interesting analytical configuration, inspired by the model of Sato (1994), is the case of a crack model growing with non constant rupture velocity. We here remain in the general configuration of an unknown average rupture velocity function, that should be regarded as the marker of the surface expansion evolution of the rupture. We now refer to this average rupture velocity as $v_{r d}$ to clearly recall that we are inside the development phase. By equating the theoretical moment function (5) and the observed one (10), we have

$$
M_{d}(t)=\frac{2 \pi}{3} \Delta \sigma a^{3}(t)=\frac{\alpha_{d}}{n_{d}+1} t^{n_{d}+1}
$$

which leads to

$$
a(t)=\int_{0}^{t} v_{r d}(u) \mathrm{d} u=\left(\frac{3 \alpha_{d}}{2 \pi \Delta \sigma\left(n_{d}+1\right)}\right)^{\frac{1}{3}} t^{\frac{n_{d}+1}{3}}
$$

and finally to the determination of the rupture velocity evolution

$$
v_{r d}(t)=\left(\frac{\alpha_{d}\left(n_{d}+1\right)^{2}}{18 \pi \Delta \sigma}\right)^{\frac{1}{3}} t^{\frac{n_{d}-2}{3}} \equiv p t^{\gamma}
$$

This derivation therefore shows that the observed power law for the moment rate function can be fully explained by rupture velocity acceleration. Rupture velocity is shown to follow a power law function with an exponent $\gamma \simeq 0.23$ and a factor $p$ inversely proportional to $\Delta \sigma^{1 / 3}$. The crack model considered here (equation (2)) can be modified to include a process zone of size $\delta_{r}$ at the tip, preventing the slip rate to diverge 


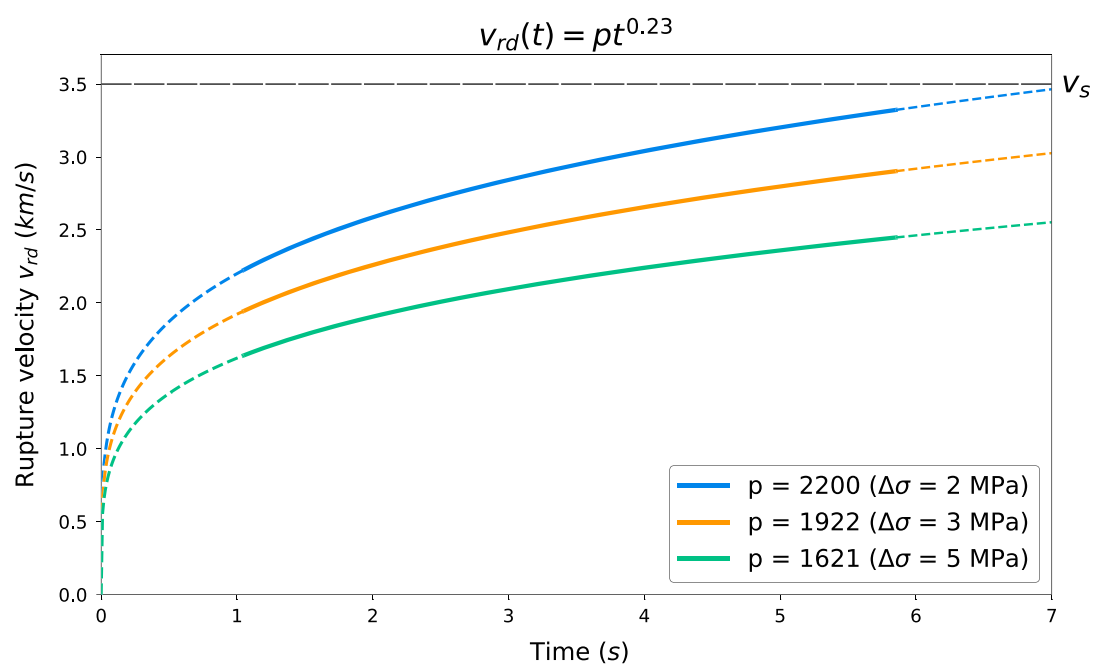

Figure 5. Rupture velocity acceleration during the development phase, as constrained by a crack model with constant stress drop. Each curve shows the equation $v_{r d}(t)=p t^{\gamma}$ for $\gamma$ fixed to the obtained value (0.23) and different values of $p$ (controlled by $\Delta \sigma$ ). Outside the time window directly constrained by the observations (between $\simeq 1$ and $\simeq 6 \mathrm{~s}$; see Figure 4), the curves are dashed; $v_{s}$ is the shear wave velocity, here fixed at a classical $3.5-\mathrm{km} / \mathrm{s}$ crustal value.

at the edges of the slipping zone. In this case, equation 2 remains valid for $r \leq a(t)-\delta_{r}$, where slip rate is maximum (and finite). This leads to a rupture velocity correlated with peak slip rate, as the slip rate calculated at $\delta r$ just behind the rupture front $\dot{u}(a(t)-\delta r, t)$ grows as $v_{r d}(t) \sqrt{a(t) / \delta r}$, or as $t^{\frac{3 \gamma+1}{2}}$ if retaining only the time dependency. This derivation is consistent with dynamic models (Bizzarri, 2012; Schmedes et al., 2010) showing that during rupture propagation, there is a positive spatial correlation between rupture velocity and peak slip rate. In contrast, the classical crack model does not lead to this correlation because peak slip rate increases as $\sqrt{t}$ while rupture velocity remains constant.

We show in Figure 5 the time evolution of $v_{r d}$ for three realistic values of $\Delta \sigma$. In the sampled part of the development phase (between $\sim 1$ and $\sim 6 \mathrm{~s}$ ) and for the realistic values of $\Delta \sigma$ shown in Figure $5, v_{r d}(t)$ gradually increases and is in a classical rupture velocity range of 1.5 to $3 \mathrm{~km} / \mathrm{s}$ (Chounet et al., 2017; Doornbos, 1982; Geller, 1976; McGuire et al., 2002; Somerville et al., 1999). This behavior may, however, be questionable for two reasons. For rupture times approaching zero (not directly sampled in the development phases), the power law predicts slow rupture velocities that have not been observed for microearthquakes (Abercrombie et al., 2017; McGuire, 2004). This requires that rupture accelerates even more abruptly in the initial instants following rupture initiation. Rupture velocity evolution also indicates that larger earthquakes, which have longer development phases, are expected to have higher local rupture velocities. However, in the magnitude range of the SCARDEC catalog, a scaling between rupture velocity and final magnitude has not been clearly observed in kinematic source analyses (Hayes, 2017; Ye et al., 2016).

The origins of the observed moment rate evolution may finally be searched in models where rupture velocity and/or stress drop have a random variability. Such models are not expected to individually follow a power law but they may collectively reproduce the average behavior of the development phase. This class of stochastic models could additionally remain self-similar, without requiring to introduce differences between small and large earthquakes.

\section{Different Behaviors Between Development Phase and Early Rupture Stage}

The development phase does not necessarily occur at early times of the rupture process. As a consequence, we do not expect to find the same time dependencies as studies focusing on how rupture starts, with reference to the earthquake origin time (Melgar \& Hayes, 2017; Meier et al., 2017). In particular, we expect the latter studies to find a less pronounced time dependency, in an average sense, due to inclusions of earthquakes with low initial moment release. In this section, we further illustrate how an average linear time dependency of the growing rupture process (Meier et al., 2017) can be approached from rupture variability rather than from intrinsic rupture properties. 


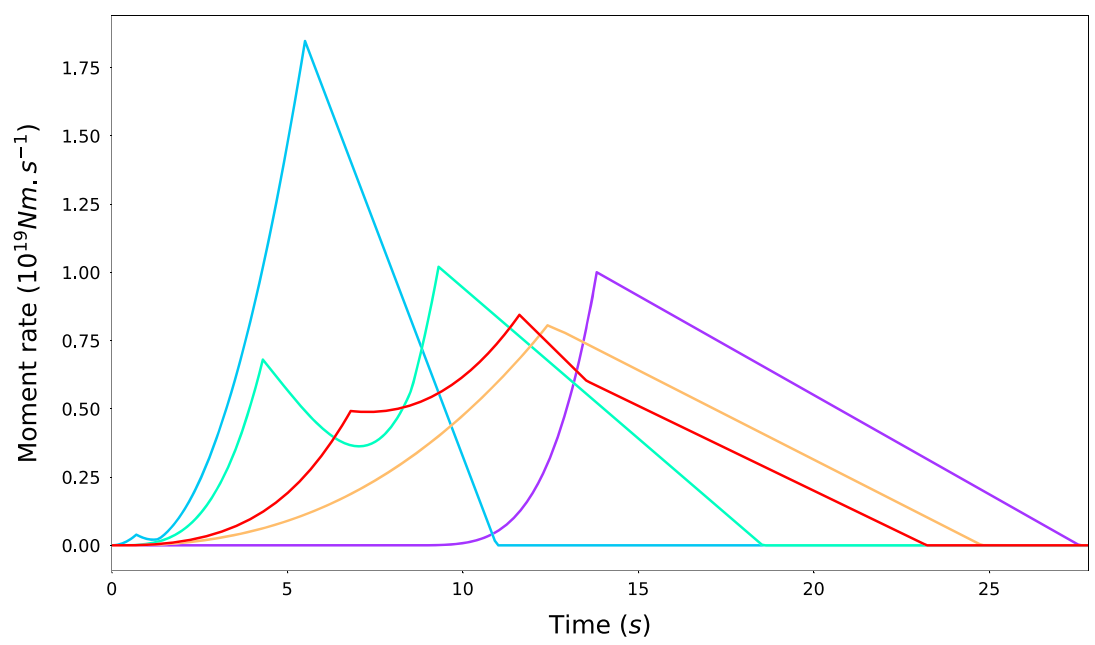

Figure 6. Examples of five synthetic STFs of $M_{w}=7$, illustrating the diversity of STF shapes in the synthetic catalog. $\mathrm{STF}=$ source time function.

To do so, we build a synthetic catalog of bimodal STFs, by summing two subevents growing both as $\dot{M}(t)=\alpha\left(t-t_{d}\right)^{n}$ where $\alpha$ and $n$ randomly vary around the observational values of $\alpha_{d}$ and $n_{d} \cdot t_{d}=0$ for the first subevent and $t_{d}$ take random values between 0 and $T_{0} / 2$ for the second subevent, where the STF total duration $T_{0}$ takes into account the observed variability around its magnitude-dependent scaling law (Courboulex et al., 2016). By also varying the relative durations (and hence moments) between the first and the second subevent, we generate a synthetic catalog with a large diversity, mimicking the main STF characteristics observed in the SCARDEC catalog: simple STFs with early development phases are obtained when the first subevent dominates, while complex ruptures, with delayed development phases, are simulated when the second subevent dominates. More details on the generation of this synthetic catalog are provided in Text S2 and Figure S2 in the supporting information, and Figure 6 shows examples of five synthetic $M_{w}=7 \mathrm{STFs}$, illustrating their large diversity.

Using the synthetic catalog, we compute the median values of the STFs at each time, as done by Meier et al. (2017) using the real STF catalogs of Ye et al. (2016), Hayes (2017), and Vallée and Douet (2016). Figure 7 shows the obtained median STFs for six magnitude bins between $M_{w}=7$ and $M_{w}=8$. No early magnitude-dependent signal is observed, as the median STFs grow in a indistinguishable way before the

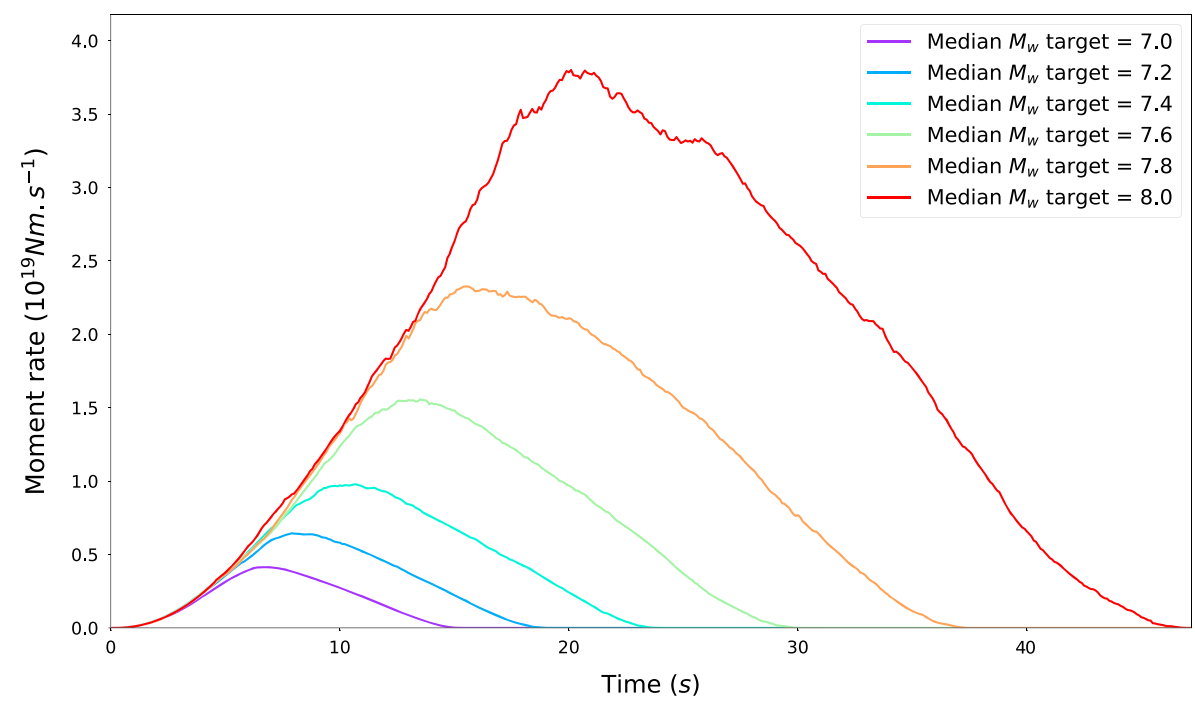

Figure 7. Median synthetic STFs from $M_{w}=7$ to $M_{w}=8$, shown in 0.2 magnitude bins. Each median STF is computed from a large number of STFs whose diversity is illustrated in Figure 6. STF = source time function. 
smallest earthquakes approach their peak. The nonlinear signal at the very beginning of each median STF is expected since all STFs have a first subevent growing with an $n$ exponent distribution centered on $n_{d}=2.68$. This early nonlinearity is also observed in the average source time functions of Melgar and Hayes (2017). Median STFs then have a flatter trend than individual subevents (equation (10)), as a result of STF diversity.

This synthetic catalog therefore illustrates how the early stages of the STFs can have average characteristics which are difficult to translate in terms of physical rupture properties. The linear behavior observed by Meier et al. (2017) quantifies how rupture starts, on average, and is useful from a practical point of view to characterize a standard STF shape. However, as we here show that this behavior can mainly result from the combined effects of nonlinear development phases and rupture diversity, its interpretation in terms of rupture dynamics must be done with care.

\section{Conclusion}

In this study, the development phase is defined as the growing phase directly preceding the peak moment rate. As such, it is not expected to behave the same way as the early stage of the seismic rupture. We here systematically extract the development phase of 2,221 STFs from the SCARDEC database in order to quantify its time evolution. For this purpose, we compute the moment acceleration (STF slope) at several moment rates, within the development phase. We first show that no magnitude-dependent signal appears, favoring a process where small and large earthquakes only differ in the duration of their development phase. Further analysis then highlights that rupture time evolution inside the development phase differs from the classical steady self-similar growth (where moment rate develops as $t^{2}$ ): the moment rate of the development phase $\dot{M}_{d}$ rather develops as $\dot{M}_{d} \propto t^{n_{d}}$ with $n_{d} \simeq 2.7 \pm 0.11$ at the $90 \%$ confidence level.

Such deviation with respect to the steady quadratic growth can be due to a combination of factors. Noncircular rupture geometry, or transient variations of stress drop and rupture velocity, may increase the time exponent of $\dot{M}_{d}$. We analytically developed one of the end-member cases, where the exponent excedence compared to the self-similar growth is purely due to monotonic rupture velocity variations. In this configuration, rupture velocity $v_{r d}$ inside the development phase is itself shown to follow a power law time function, with $v_{r d} \propto t^{0.2}$. Due to this low exponent, $v_{r d}$ is expected to quickly reach classical rupture velocities $(>1 \mathrm{~km} / \mathrm{s}$ ) and then to increase only moderately in the sampled part of the development phase (between $\sim 1$ and $\sim 6 \mathrm{~s}$ ) More generally, even if the observed time dependency of the development phase may have several causes, it should give a new observational constraint to assess the realism of dynamic rupture scenarios.

Acknowledgments

We thank Pascal Bernard, Claudio Satriano, Alexandre Schubnel, and Hideo Aochi for constructive remarks on this work. Fruitful discussions with Men-Andrin Meier improved our analysis of median STFs and the generation of the synthetic catalog. Constructive reviews provided by Gavin Hayes and an anonymous reviewer helped us to improve the original manuscript. We are grateful to the FDSN, for the public availability of the broadband seismograms of the global network, and to the IRIS Data Center and IPGP data center for easy access to the data. SCARDEC database is available at http://scardec.projects. sismo.ipgp.fr/ website. This study contributes to the IdEx Université de Paris ANR-18-18-IDEX-0001.

\section{References}

Abercrombie, R. E., Poli, P., \& Bannister, S. (2017). Earthquake directivity, orientation, and stress drop within the subducting plate at the Hikurangi Margin, New Zealand. Journal of Geophysical Research: Solid Earth, 122, 10,176-10,188. https://doi.org/10.1002/ 2017JB014935

Aki, K. (1967). Scaling law of seismic spectrum. Journal of Geophysical Research, 72(4), 1217-1231.

Allmann, B. P., \& Shearer, P. M. (2009). Global variations of stress drop for moderate to large earthquakes. Journal of Geophysical Research, 114, B01310. https://doi.org/10.1029/2008JB005821

Archuleta, R. J., \& Ji, C. (2016). Moment rate scaling for earthquakes $3.3 \leq \mathrm{m} \leq 5.3$ with implications for stress drop. Geophysical Research Letters, 43, 12,004-12,011. https://doi.org/10.1002/2016GL071433

Beroza, G. C., \& Ellsworth, W. L. (1996). Properties of the seismic nucleation phase. Tectonophysics, 261(1-3), $209-227$.

Beroza, G. C., \& Spudich, P. (1988). Linearized inversion for fault rupture behavior: Application to the 1984 Morgan Hill, California, earthquake. Journal of Geophysical Research, 93(B6), 6275-6296.

Bilek, S., Lay, T., \& Ruff, L. (2004). Radiated seismic energy and earthquake source duration variations from teleseismic source time functions for shallow subduction zone thrust earthquakes. Journal of Geophysical Research, 109, B09308. https://doi.org/10.1029/ 2004JB003039

Bizzarri, A. (2012). Rupture speed and slip velocity: What can we learn from simulated earthquakes? Earth and Planetary Science Letters, $317,196-203$.

Brune, J. N. (1970). Tectonic stress and the spectra of seismic shear waves from earthquakes. Journal of Geophysical Research, 75(26), 4997-5009.

Causse, M., Dalguer, L., \& Mai, P. M. (2013). Variability of dynamic source parameters inferred from kinematic models of past earthquakes. Geophysical Journal International, 196(3), 1754-1769.

Chounet, A., \& Vallée, M. (2018). Global and interregion characterization of subduction interface earthquakes derived from source time functions properties. Journal of Geophysical Research: Solid Earth, 123, 5831-5852. https://doi.org/10.1029/2018JB015932

Chounet, A., Vallée, M., Causse, M., \& Courboulex, F. (2017). Global catalog of earthquake rupture velocities shows anticorrelation between stress drop and rupture velocity. Tectonophysics, 733, 148-158.

Colombelli, S., Zollo, A., Festa, G., \& Picozzi, M. (2014). Evidence for a difference in rupture initiation between small and large earthquakes. Nature Communications, 5, 3958. 
Courboulex, F., Vallée, M., Causse, M., \& Chounet, A. (2016). Stress-drop variability of shallow earthquakes extracted from a global database of source time functions. Seismological Research Letters, 87(4), 912-918.

Dahlen, F. (1974). On the ratio of p-wave to s-wave corner frequencies for shallow earthquake sources. Bulletin of the Seismological Society of America, 64(4), 1159-1180.

Das, S., \& Kostrov, B. (1983). Breaking of a single asperity: Rupture process and seismic radiation. Journal of Geophysical Research, 88(B5), 4277-4288.

Denolle, M. A., \& Shearer, P. M. (2016). New perspectives on self-similarity for shallow thrust earthquakes. Journal of Geophysical Research: Solid Earth, 121, 6533-6565. https://doi.org/10.1002/2016JB013105

Doornbos, D. (1982). Seismic moment tensors and kinematic source parameters. Geophysical Journal International, 69(1), 235-251.

Dunham, E. M., Favreau, P., \& Carlson, J. (2003). A supershear transition mechanism for cracks. Science, 299(5612), 1557-1559.

Geller, R. J. (1976). Scaling relations for earthquake source parameters and magnitudes. Bulletin of the Seismological Society of America, 66(5), 1501-1523.

Hayes, G. P. (2017). The finite, kinematic rupture properties of great-sized earthquakes since 1990. Earth and Planetary Science Letters, $468,94-100$.

Houston, H. (2001). Influence of depth, focal mechanism, and tectonic setting on the shape and duration of earthquake source time functions. Journal of Geophysical Research, 106(B6), 11,137-11,150.

Houston, H., Benz, H. M., \& Vidale, J. E. (1998). Time functions of deep earthquakes from broadband and short-period stacks. Journal of Geophysical Research, 103(B12), 29,895-29,913.

Kanamori, H., \& Anderson, D. L. (1975). Theoretical basis of some empirical relations in seismology. Bulletin of the Seismological Society of America, 65(5), 1073-1095.

Kostrov, B. (1964). Self-similar problems of propagation of shear cracks. PMM, 28, 889-898.

Madariaga, R. (1976). Dynamics of an expanding circular fault. Bulletin of the Seismological Society of America, 66(3), 639-666.

Mai, P., Somerville, P., Pitarka, A., Dalguer, L., Song, S., Beroza, G., et al. (2006). On scaling of fracture energy and stress drop in dynamic rupture models: Consequences for near-source ground-motions. Geophysical Monograph-American Geophysical Union, $170,283$.

McGuire, J. J. (2004). Estimating finite source properties of small earthquake ruptures. Bulletin of the Seismological Society of America, 94(2), 377-393.

McGuire, J. J., Zhao, L., \& Jordan, T. H. (2002). Predominance of unilateral rupture for a global catalog of large earthquakes. Bulletin of the Seismological Society of America, 92(8), 3309-3317.

Meier, M.-A., Ampuero, J., \& Heaton, T. H. (2017). The hidden simplicity of subduction megathrust earthquakes. Science, 357(6357), $1277-1281$.

Melgar, D., \& Hayes, G. P. (2017). Systematic observations of the slip pulse properties of large earthquake ruptures. Geophysical Research Letters, 44, 9691-9698. https://doi.org/10.1002/2017GL074916

Melgar, D., \& Hayes, G. P. (2019). Characterizing large earthquakes before rupture is complete. Science Advances, 5(5), eaav2032.

Nielsen, S., \& Madariaga, R. (2003). On the self-healing fracture mode. Bulletin of the Seismological Society of America, 93(6), 2375-2388.

Olson, E. L., \& Allen, R. M. (2005). The deterministic nature of earthquake rupture. Nature, 438(7065), 212.

Sato, T. (1994). Seismic radiation from circular cracks growing at variable rupture velocity. Bulletin of the Seismological Society of America, 84(4), 1199-1215.

Schmedes, J., Archuleta, R. J., \& Lavallée, D. (2010). Correlation of earthquake source parameters inferred from dynamic rupture simulations. Journal of Geophysical Research, 115, B03304. https://doi.org/10.1029/2009JB006689

Somerville, P., Irikura, K., Graves, R., Sawada, S., Wald, D., Abrahamson, N., et al. (1999). Characterizing crustal earthquake slip models for the prediction of strong ground motion. Seismological Research Letters, 70(1), 59-80.

Tanioka, YuichiroandRuff (1997). Source time functions. Seismological Research Letters, 68(3), 386-400.

Uchide, T., \& Ide, S. (2010). Scaling of earthquake rupture growth in the Parkfield area: Self-similar growth and suppression by the finite seismogenic layer. Journal of Geophysical Research, 115, B11302. https://doi.org/10.1029/2009JB007122

Vallée, M. (2013). Source time function properties indicate a strain drop independent of earthquake depth and magnitude. Nature Communications, 4, 2606

Vallée, M., Charléty, J., Ferreira, A. M., Delouis, B., \& Vergoz, J. (2011). Scardec: A new technique for the rapid determination of seismic moment magnitude, focal mechanism and source time functions for large earthquakes using body-wave deconvolution. Geophysical Journal International, 184(1), 338-358.

Vallée, M., \& Douet, V. (2016). A new database of source time functions (STFs) extracted from the scardec method. Physics of the Earth and Planetary Interiors, 257, 149-157.

Ye, L., Lay, T., Kanamori, H., \& Rivera, L. (2016). Rupture characteristics of major and great (Mw $\geq 7.0$ ) megathrust earthquakes from 1990 to 2015: 1. Source parameter scaling relationships. Journal of Geophysical Research: Solid Earth, 121, 826-844. https://doi.org/10. 1002/2015JB012426

Zhang, G., Vallée, M., Shan, X., \& Delouis, B. (2012). Evidence of sudden rupture of a large asperity during the 2008 Mw7.9 wenchuan earthquake based on strong motion analysis. Geophysical Research Letters, 39, L17303. https://doi.org/10.1029/2012GL052516 